\title{
Gastroscope-Facilitated Endotracheal Intubation During ERCP: When Is the Best Time to GETA (Big) MAC?
}

\author{
Tilak Shah ${ }^{1,2} \cdot$ Stefan lanchulev ${ }^{3}$
}

Published online: 9 July 2020

(c) This is a U.S. Government work and not under copyright protection in the US; foreign copyright protection may apply 2020

\section{Gastroenterologist's Perspective}

Endoscopic retrograde cholangiopancreatography (ERCP), once primarily a diagnostic procedure, has evolved considerably over the last several decades. Since noninvasive imaging tests such as magnetic resonance cholangiopancreatography (MRCP) have largely replaced diagnostic ERCP, the procedure now serves as a platform for multiple complex and often time-consuming therapeutic maneuvers. Though the procedure was once routinely performed under moderate sedation, longer cases require a higher cumulative dose of opiates and benzodiazepines in order to maintain moderate sedation and thus pose an increased risk of sedation-related adverse events. As a result, most centers in the USA have abandoned moderate sedation in favor of anesthesiologistdirected deep sedation or general anesthesia. Thus, the responsibility for safe and effective sedation during ERCP now lies largely in the hands of the anesthesiologist.

Deep sedation under monitored anesthesia care (MAC) may be faster and less resource-intensive than general anesthesia with endotracheal intubation (GETA) [1]. Several studies have demonstrated that the risk of serious complications is low when MAC is utilized for ERCP in healthier, non-obese patients $[2,3]$. On the other hand, GETA provides maximal airway protection, particularly when ERCP is performed in the prone position or with a full stomach, and may increase procedure success rates [3]. In a randomized trial of

Tilak Shah

Tilak.Shah@va.gov

1 Division of Gastroenterology and Hepatology, Central Virginia VA Healthcare System, 111N, 1201 Broad Rock Blvd, Richmond, VA 23224, USA

2 Division of Gastroenterology and Hepatology, Virginia Commonwealth University, Richmond, VA, USA

3 Department of Anesthesia, Central Virginia VA Healthcare System, 111N, 1201 Broad Rock Blvd, Richmond, VA 23224, USA
200 patients at high risk for sedation-related complications undergoing ERCP, significant respiratory events occurred in 40\% more patients with MAC compared with GETA, with $10 \%$ of MAC cases required conversion to GETA [4]. Thus, the published literature supports GETA for higher complexity patients, and is likely the optimal option for longer and more complex procedures, even in healthy patients $[4,5]$. MAC may be a safe and more efficient option for low-complexity cases in patients with minimal comorbidities.

In this issue of Digestive Diseases and Sciences, Barakat and colleagues describe their single-center experience with endoscopist-facilitated endotracheal intubation (ETI) using an ultra-slim gastroscope when required for hypoxia or retained food during MAC-assisted ERCP [6]. Their rationale for this approach is that when salvage ETI is required during MAC-assisted ERCP performed in the prone position, multiple steps are required: (i) a gurney must be brought into the fluoroscopy room, (ii) the semiprone patient is transferred to the gurney and placed in the supine position in order to perform ETI, and (iii) following ETI, the patient is again repositioned to the prone position. Of the 3409 patients who underwent ERCP (2014-2019), MAC sedation was utilized in 1841 (54\%), and 42 (2.3\%) required ETI. Among the 42 patients, endoscopist-facilitated ETI was successfully accomplished in 16 patients (all due to hypoxia). Most cases were performed by one endoscopist who was self-trained in the technique; a few were performed by a junior faculty member who had observed the technique during fellowship training. Notably, not all therapeutic endoscopists at the institution utilized this technique.

Bronchoscopy-facilitated ETI using a standard 4.0-6.0 mm device is a well-established maneuver, particularly in difficult airway situations [7]. Since the inner diameter of the smallest adult endotracheal tube (ETT) is $6 \mathrm{~mm}$, it should also readily accommodate an ultra-slim gastroscope (outer diameter of $5.4 \mathrm{~mm}$ ). From a technical standpoint, the skillset to perform gastroscope-facilitated ETI (G-ETI) is already within the therapeutic endoscopist's toolbox, since 
they routinely pass over-tubes into the esophagus over a gastroscope so as to manage food impactions and for foregut suturing. The authors are to be commended for demonstrating the utility of G-ETI in a real-world high-stakes environment (hypoxia during MAC ERCP). One could envision G-ETI as a useful technique for non-ERCP cases when an anesthesiologist is not immediately available (e.g.,- - aspiration or large volume of clot in the stomach during an upper endoscopy performed under moderate sedation). Yet, for endoscopists who wish to incorporate this procedure into their practice, a few caveats with regards to logistics, training, credentialing, and medicolegal liability are worth considering. Although transferring a patient to the supine position takes time, so does switching from a side-viewing ERCP scope to an ultra-slim gastroscope; when ERCP is performed in the supine position G-ETI may take longer to initiate than standard ETI. Furthermore, since ETI carries its own subtle nuances regarding anatomic landmarks and cuff placement, expertise in esophageal intubation does not necessarily confer expertise in ETI. From the standpoint of legal liability, a plaintiff could argue that an endoscopist was not adequately supervised if there was a nurse anesthetist but not an anesthesiologist present in the room. Due to these concerns, credentialing committees may justifiably require an endoscopist to demonstrate training in ETI before granting privileges to perform G-ETI. But given the ever-increasing trend of performing ERCPs under GA, endoscopists may find they lack the volume required to maintain this important skillset. Indeed, even in a center that performs about 680 ERCPs a year with a high percentage $(>50 \%)$ under $\mathrm{MAC},<3 \%$ required conversion to GA, and only $16 \mathrm{G}$-ETIs were performed in 5 years. In order to obtain and maintain credentials in ETI, endoscopists may therefore need to perform a certain number of supervised elective ETIs, supplemented by periodic simulator training in G- ETI. Some endoscopists may find this effort worthwhile to keep this "trick" up their sleeve during MAC ERCP, but for many others, the commitment may feel like a fool's errand.

\section{Anesthesiologist's Perspective}

ETI is no longer solely in the domain of anesthesiology having crossed the borders into emergency medicine, intensive care, and otolaryngology. It is no surprise to us that a gastroenterologist skillful with the endoscope can become proficient in G-ETI and use it as a rescue procedure.

From the anesthesiologist's point of view, GETA is the preferred approach to manage ERCPs, particularly in patients with comorbidities. Given the length of the procedure, semi-prone position, and aspiration risk, GETA is the least anxiety-provoking approach for the anesthesiologist [8]. Yet in this study, 50\% of ERCPs were performed under MAC with deep sedation with a very low incidence of adverse events $(<2 \%)$ [6]. The tools available to anesthesiologists and the availability of fast on/off medications like propofol have significantly decreased MAC-related complications. Still, two types of complications are more common with MAC, namely desaturation due to hypopnea and aspiration. Both types of complications require additional interventions and are associated with increased resource utilization.

With regard to hypoventilation and associated hypoxemia, the borderline between deep sedation and general anesthesia is easily blurred. Despite the definition of deep sedation, most patients undergoing ERCP are in de facto general anesthesia without paralysis or ETI. There are a myriad of options for these patients, including, but not limited to: ETI, laryngeal mask airway (LMA; there are LMAs specifically designed to accommodate an endoscope), high flow nasal cannulas, and simple maneuvers such as jaw thrust, which any can help to safely manage the patient to the completion of ERCP. In the face of other options, we must weigh the choice to use G-ETI against the risk of airway damage that can occur during the intubation process.

The only safe way to prevent and manage aspiration, from an anesthesiology viewpoint, is by intubating the trachea from the onset, i.e., to perform the procedure under GETA. Healthcare teams must weigh the purported time savings against patient safety and healthcare resource utilization. Such patients are usually admitted, at least overnight, and often require antibiotics and additional resources. The question becomes more of how to predict which patient will aspirate. Although 500,000 ERCPs are performed each year in the USA, the incidence of witnessed aspiration is $<1 \%$ [9], we can assume that the processes already established in individual institutions are working well. More recently, ultrasound examination of the stomach has been reported to provide insight and possibly guidance with the decision to use MAC versus GETA [10].

The adoption of a team model in the endoscopy center is an important concept, where everyone present for the procedure is part of the same team. This improves communication and delineation of care. Under such circumstances, one can expect that rescue management of the airway is discussed prior to the procedure. Such discussions will alleviate anesthesiologists' anxiety as they typically care for multiple patients simultaneously, and aspiration under deep sedation in prone or semi-prone position will require swift actions in managing the patient's airway. If the endoscopist is comfortable with G-ETI, has the resources available, and a nurse anesthetist present for backup, the endoscopist could secure the airway using G-ETI since all the planning and discussions have already taken place. The advantage of using an endoscope to intubate in such rescue maneuvers is the ability to suction out the aspirated contents. It is prudent to 
have an understanding that even such endoscopist-driven intubations can encounter difficulties and may require an experienced anesthesiologist to handle them. For instance, endoscopists do not routinely use muscle relaxants. Transitioning to a team approach, including endoscopist G-ETI, to rescue-manage the airway is a valuable planning concept to safeguard patient safety. As is true in much of medicine, preventive actions are more efficient overall than rescue efforts.

Funding Dr. Shah receives funding from the American Society for Gastrointestinal Endoscopy (ASGE) and McGuire Research Institute (MRI). The content is solely the responsibility of the authors and does not necessarily represent the official views of the ASGE or MRI.

\section{Compliance with Ethical Standards}

Conflict of interest Dr. Shah served on a research advisory board for CSA Medical, Inc and receives research support from Abbvie. Dr. Ianchulev has no financial conflicts to report.

\section{References}

1. Perbtani YB, et al. Impact of endotracheal intubation on interventional endoscopy unit efficiency metrics at a tertiary academic medical center. Am J Gastroenterol. 2016;111:800-807.

2. Barnett SR, et al. Deep sedation without intubation for ERCP is appropriate in healthier, non-obese patients. Dig Dis Sci. 2013;58:3287-3292. https://doi.org/10.1007/s10620-013-2783-x.

3. Liang CM, et al. A comparison between non-sedation and general endotracheal anesthesia for retrograde endoscopic common bile duct stone removal: a tertiary center experience. Biomed $J$. 2019;42:131-136.

4. Smith ZL, et al. A randomized controlled trial evaluating general endotracheal anesthesia versus monitored anesthesia care and the incidence of sedation-related adverse events during ERCP in highrisk patients. Gastrointest Endosc. 2019;89:855-862.

5. Cotton PB, et al. Grading the complexity of endoscopic procedures: results of an ASGE working party. Gastrointest Endosc. 2011;73:868-874.

6. Barakat M, Angelotti T, Banerjee S. Use of an ultra-slim gastroscope to accomplish endoscopist-facilitated rescue intubation during ERCP: a novel approach to enhance patient and staff safety. Dig Dis Sci. (Epub ahead of print). https://doi.org/10.1007/s1062 0-020-06360-w.

7. Apfelbaum JL, et al. Practice guidelines for management of the difficult airway: an updated report by the American Society of Anesthesiologists Task Force on Management of the Difficult Airway. Anesthesiology. 2013;118:251-270.

8. Stone AB, et al. A medicolegal analysis of malpractice claims involving anesthesiologists in the gastrointestinal endoscopy suite (2007-2016). J Clin Anesth. 2018;48:15-20.

9. Cappell MS, Friedel DM. Stricter national standards are required for credentialing of endoscopic-retrograde-cholangiopancreatography in the United States. World J Gastroenterol. 2019;25:3468-3483.

10. Perlas A, et al. Ultrasound assessment of gastric content and volume. Anesthesiology. 2009;111:82-89.

Publisher's Note Springer Nature remains neutral with regard to jurisdictional claims in published maps and institutional affiliations. 